Fecha de recepción: marzo 2020 Fecha de aceptación: abril 2020 Versión final: mayo 2020

\section{El concepto de Imagen en la} nueva normalidad

María Pía Estebecorena ${ }^{(1)}$

Resumen: El concepto de Pandemia mundial ha forzado a cambiar la comunicación interpersonal en una forma abrupta. Dentro de esta adaptación, el concepto de Imagen como tal está elaborando su reformulación.

Hoy más que nunca Imagen es percepción y comunicación. En este trabajo se podrá ver como la pregunta sobre qué es la Imagen se ha reformulado casi por sí sola. Como especialistas en Imagen, hoy aparecen frente a nosotros aspectos de la vida de los otros que antes no veíamos, necesidades nuevas a partir de la imagen personal por parte de los clientes, que piden incorporar un nuevo aprendizaje sobre su propia Imagen y como ésta comunica a los demás.

Hoy los clientes perciben que su Personal Branding es clave dentro de la comunicación en esta nueva normalidad. La resignificación del concepto Imagen ha iniciado un cambio sin retorno planteando un nuevo desafío en la profesión.

Palabras clave: Nueva normalidad - COVID19 - Personal Branding - Marca Personal Imagen - Digital - Resiliencia - Percepción - Neuroliderazgo - Neurobusiness - Neuroimagen.

[Resúmenes en inglés y portugués en las páginas 59-60]

(1) María Pía Estebecorena es Lic. en Ciencia Política, Asesora de Imagen. Cuenta con Posgrados en Psicología Positiva, Estrategia, Geopolítica, Retail Marketing \& Loyalty (CRM). Certificada internacionalmente en Incubadora de Negocios por la Universidad de Salamanca y Doinglobal, Sillicon Valley. Es Point of You Trainer dentro del área de Coaching. Actualmente es parte del Directorio Internacional de AICI como VP Business Development. Fue Membership Chair of Latin America \& Spain for AICI Global, Past President de AICI Argentina por dos períodos y fundadora del Capitulo. Trabajó como Directora de Negocios y Product Manager por más de 16 años para: Falabella, Walmart, Tiendas Paris de Chile y estuvo a cargo del start up de Elektra de Argentina del Grupo Salinas de México. En el año 2011 UNESCO la distinguió como Embajadora de Paz. Especialista en Personal Branding, Management and Professional Image. Speaker internacional, dando capacitaciones por todo Latinoamérica, USA e India. En el 2017 obtuvo la Certificación Internacional en Imagen por AICI Internacional, AICI CIC y en 2019 la Certificación AICI CIP. A cargo de CEImagen, Capacitación y Entrenamiento en Imagen y Personal Branding desde 2001. 
Las crisis interrumpen el desarrollo pasivo de nuestras vidas y nos hacen más conscientes de lo que realmente importa, sacándonos de nuestra zona de confort. Nunca más que hoy esta frase cobra verdadero sentido.

Desde hace casi 20 años he estudiado el concepto de Imagen y éste ha ido variando con el tiempo ajustándose cada vez más a una definición más amplia a medida que las necesidades y la comunicación iban cambiando

Este concepto que comenzó hace 20 años a desarrollarse en el mundo con mas fuerza y en Argentina apenas iniciaba, describía las formas de vestimenta, la etiqueta social y casi sólo comprendía a la imagen femenina. Ese momento dista mucho de parecerse al actual y curiosamente el concepto no varió tanto por la imagen en sí. Por el contrario, la variación impacta sobre el cambio de dos factores principales: la comunicación y los valores.

En este trabajo intentaré mostrar esta transformación del concepto a medida que avanzaba en importancia e impacto.

\section{Comunicación e Imagen}

A lo largo de los últimos 20 años el giro en el concepto de comunicación ha sido impactante. Según estudios ${ }^{1}$ el lapso de atención en cuanto a la capacidad sostenida y focalizada en un mismo estímulo, era en promedio en el año 2000 de entre 15 a 12 segundos. Actualmente se encuentra entre los 8 y 5 segundos. Esto significa que los primeros segundos son claves para establecer una buena comunicación.

Esta comunicación incluye lo que percibimos a través del lenguaje no verbal, los colores, el estilo personal y las actitudes a través del accionar. También lo que trasciende a nuestra persona, pero nos pertenece como las redes sociales en las que compartimos con los demás.

Si el mensaje no es suficientemente atractivo, la atención baja y desaparece. Solo contamos con 8 segundos para crear el efecto halo positivo.

Efectivamente este cambio en la comunicación es un impacto directo al concepto de Imagen y una transformación que apareció junto con su significado. Hay Imagen es percepción. Siempre lo fue, solo que lo que ha cambiado es la consciencia de quien percibe y la cantidad de exposición que hoy tiene nuestra imagen.

De acuerdo al estudio del Dr. George Markowsky², la transmisión de la información recibida a través de nuestros cinco sentidos es de 11 millones de bits por segundo y es captada en forma inconsciente, mientras que la información que nos llega en forma consciente es de 50 bits, tal como podemos ver en este gráfico, diferenciado por cada sentido. 


\begin{tabular}{|c|c|}
\hline \multicolumn{2}{|c|}{ Tasa de transmisión de la información percibida por los sentidos } \\
\hline \multicolumn{1}{|c|}{ Sentido } & Bits por segundo \\
\hline Vista & $10,000,000$ \\
\hline \multicolumn{1}{|c|}{ Tacto } & $1,000,000$ \\
\hline Audición & 100,000 \\
\hline Olfato & 100,000 \\
\hline Gusto & 1,000 \\
\hline $\begin{array}{l}\text { Total información ingresada a través de los sentidos } \\
\text { (Bits por segundo) }\end{array}$ & 11.201 .000 \\
\hline $\begin{array}{l}\text { Información que llega hasta nivel consciente (Bits por } \\
\text { segundo) }\end{array}$ & 50 \\
\hline $\begin{array}{l}\text { Información que llega a nivel consciente / total } \\
\text { información ingresada a través de los sentidos (\%) }\end{array}$ & $0,000454545 \%$ \\
\hline Fuente: George Markowsky, "Information Theory". Encyclopædia Britannica Online \\
\hline
\end{tabular}

\section{La imagen es invisible a los ojos}

A partir de todos los datos podemos concluir que hoy el concepto de imagen en 20 años ha cambiado en cuanto a su percepción. Hoy hablar de imagen ya no refiere a la forma de vestir, el outfit o los accesorios. No solamente. Hablar de imagen es incluir todos estos aspectos en un pensamiento integral de ese concepto. Decir que la imagen es invisible a los ojos, es decir, que, además, es percibida por otros canales de comunicación que la nutren y del cual forman parte.

Estamos hablando de la integración de los aspectos que comprenden la personalidad, la psicología y la energía personal lo cual se traduce para las empresas y las relaciones laborales y personales en el manejo de las habilidades blandas de la persona.

Con el paso del tiempo, en un lapso de 10 años, las compañías que antes requerían solamente entrenamiento en imagen externa, pasaron a requerir un $63 \%$ mas entrenamiento en habilidades blandas ${ }^{3}$.

A partir del comienzo de la 4ta Revolución en la era digital el criterio de selección pasó a darle mayor importancia a este tipo de concepto de imagen, comprendida en una idea global, integral $360^{\circ}$ en la integración de todos los componentes de la persona.

La tendencia en las compañías a nivel global es enfocarse al momento de la selección en estos aspectos, que describo a continuación. En todas, la imagen es un factor determinante, evaluada en sus diferentes formas.

Podemos decir que los aspectos por los cuales la imagen personal será destacada y valorizada dentro de la nueva normalidad, son aquellos que compone el Neuroliderazgo y que influye en el Neuromanagement profesional ${ }^{4}$ : 
- Gestión de conflictos

- Gestión del tiempo

- Manejo del stress laboral

- Productividad personal

- Inteligencia emocional

- Habilidades de comunicación

- Gestión de cambio

\section{Hacia un concepto de Imagen $360^{\circ}$}

Quienes trabajamos como especialistas en Imagen, además de las nuevas corrientes dentro del mundo laboral que se han ido incorporando estos años, debemos dedicar un entrenamiento en nuevos aspectos dentro del campo profesional.

Estos aspectos aparecen en escena cuando la visión social cambia su mirada a temas como la Diversidad en todas sus formas y la tolerancia comienza a ser un valor destacado sobre el cual podemos ayudar como Asesores a la deconstrucción y reconstrucción en el entrenamiento sobre estos temas.

Cuando mencionamos Diversidad nos referimos a un amplio tema, un gran titular que abre un capítulo aparte. Dentro de las organizaciones, corporaciones y/o industrias la diversidad puede significar hablar de:

- Género

- Sexual

- Etaria

- Cultural

- Social

- Educativa

- Ideológica

En estas organizaciones están comprendidas desde las empresas hasta los foros sociales, ONG y asociaciones profesionales que ya han incorporado la diversidad dentro de su orden estructural. El banco HSBC en Argentina es un ejemplo de la integración de diversidad de género, etaria y cultural ${ }^{5}$. Walmart desde hace más de 15 años trabaja con diversidad etaria empleando a mayores de 50 años en todo el mundo. Estos son apenas unos ejemplos de cómo a medida que la sociedad incorpora nuevos conceptos, van modificando valores y con ellos el concepto de imagen acompaña esta evolución.

Un gran desafío para quienes componen el sector de expertos en imagen es incorporar estos entrenamientos a su perfil profesional con capacitaciones.

Hoy un Asesor especializado en áreas laborales requiere de conocer además de oratoria, la importancia de trabajar los sesgos en las corporaciones o al menos debería poder identificarlos en el discurso de sus clientes preocupados por su imagen profesional. 
Es indudable que todos estos aspectos, hoy son inseparables de lo que entendemos por imagen, por tanto, un Asesor debe poder ofrecer respuestas a sus clientes dentro de un cambio tan profundo en lo social como el actual.

Desde AICI destacamos tres aspectos relevantes: Apariencia. Comportamiento. Comunicación.

Esta es la concepción integral de la imagen personal. La necesidad de este cambio global requiere de nuevas respuestas acorde a los tiempos y necesidades del cliente. Los valores apreciados en una Directora o un Gerente se enfocan en un sólido entrenamiento de sus capacidades humanas y de una trayectoria ética y transparente. Son valores absolutamente distantes de los que se esperaba encontrar en el mismo perfil en el año 2000.

\section{Perspectivas en Imagen}

La capacidad de supervivencia estará dada una vez más por la velocidad de adaptación que tengas los profesionales en Imagen a estas nuevas condiciones. Muhammad Yunus, Premio Nobel de la Paz, emprendedor y banquero indio declaró a la crisis de COVID19 como una de las grandes oportunidades para generar un nuevo mundo.

"Si queremos aprovechar esta oportunidad para crear un nuevo mundo, debemos construir nuevos caminos basados en nuevas formas de pensar. Volver al viejo mundo bajo ningún concepto es una opción"6.

Repensar la función de nuestra profesión significa aceptar los nuevos paradigmas que acompañen en las nuevas problemáticas que enfrenta el cliente. Quienes nos dedicamos a la formación de profesionales en el área tendremos la tarea de entrenar estas nuevas perspectivas para abrir posibilidades de empleo diferentes. Un mundo que ya no luce diseños, tendencias, donde su imagen visual se ha reducido a una foto o una transmisión digital, el cual esta nueva normalidad atraviesa también al mismo código de comportamiento social a partir del momento en que el distanciamiento social se ha impuesto.

Estas nuevas perspectivas requieren una nueva forma de pensar el concepto Imagen que hoy parece no encontrar aún su nuevo camino. La pregunta a hacerse es ¿para qué cliente y con cuáles necesidades elaborar una propuesta de asesoramiento en imagen o de entrenamiento? En este inicio de la nueva normalidad las necesidades de clientes pasan por manejar en forma eficaz la alta exposición de su imagen. Aspectos técnicos de aparición en cámara como colores, proporciones. Pero los requerimientos más destacados son los que refieren a las habilidades blandas y el entrenamiento de nuevas habilidades que están comprendidas en la imagen interna.

Los entrenamientos en Marca Personal hoy lideran los requerimientos en asesorías, porque la demanda de empleo se acortó, se modificó y registra un pico de movilidad muy grande. Sucede lo mismo con los requerimientos en Imagen Política.

Hay que tener en cuenta que vamos ingresando hacia una Economía de la atención. Dice Herbert Simon: 
En un mundo rico en información, la riqueza de información significa una escasez de otra cosa: la escasez de lo que sea que la información consuma. La información que consume es bastante obvia: consume la atención de sus destinatarios. Por lo tanto, una gran cantidad de información crea una pobreza de atención y la necesidad de asignar esa atención eficientemente entre la abundancia de fuentes de información que podrían consumirla ${ }^{7}$.

Simon es uno de los primeros en abordar en los años 70 este tema hoy tan vigente. En un contexto en donde la atención es un valor preciado y tomando en cuenta las referencias sobre el decrecimiento de la atención en los últimos 20 años tal como está mencionado al inicio de este artículo, la necesidad creciente de un buen manejo integral del Personal branding se impone como una herramienta fundamental dentro de la perspectiva profesional para los expertos en imagen.

En esta economía la regla es ganar visibilidad. A su vez, esta visibilidad tiene que ser corta, directa, concreta y bien comunicada.

Esto incluye la capacidad de crear nuevas estrategias para clientes en canales de comunicación diferentes a lo que pensamos como Imagen Externa. Hoy más que nunca antes lo externo está supeditado y condicionado como un elemento de comunicación y expresión del yo, refleja valores, pensamientos (Pañuelos verde y celeste en Argentina son un ejemplo del poder del pensamiento aplicado a la Imagen). La Imagen interna encuentra expresión en lo externo uno de sus canales de expresión.

\section{Ecosistema emprendedor y nueva normalidad}

A modo de conclusión este trabajo plantea un desafío para los profesionales en Imagen a partir de la evolución del concepto.

- Incorporar nuevas perspectivas para asistir nuevos requerimientos.

- Poder enfocar el negocio pensando dentro de esta nueva normalidad con capacidad de reinvención es mandatario. Perspectiva de negocio como un ecosistema emprendedor

- Reencontrar un nuevo nicho en un mercado digitalizado

Son los expertos en Imagen los que están encargados de repensar y reformular esta nueva era en un contexto volátil y cambiante a una gran velocidad. Como emprendedores, en un contexto de negocio el desafío es pensar en términos colaborativos, de alianzas con colegas para lograr que el Ecosistema emprendedor se desarrolle según la ética profesional que plasmemos en nuestro propio Personal Branding.

Cada profesional vuelca su experiencia dentro del ecosistema. Es un concepto que ha ido avanzando muy velozmente, se adapta muy bien a los requerimientos actuales y es necesariamente transparente hacia el cliente. Pensar el negocio desde la perspectiva de Ecosistema implica una adaptación a un formato diferente. El cambio tendrá que ocurrir de 
todas formas y el ecosistema plantea una buena solución cuando los requerimientos son múltiples desde el cliente.

Adaptarse a los nuevos desafíos implica evolucionar a una forma de ver y trabajar dentro de esta profesión, adaptada a la nueva normalidad

\title{
Notas
}

1. BBC Londres - Instituto Canadiense del Estudio de la Salud (CIHR) 2015 /2018.

2. George Markowsky, Information Theory. Encyclopedia Britannica Online 2017.

3. Investigación de CEimagen para AICI Global Business sobre Consultores en Latinoamérica y España 2019/2020.

4. Advancing the Science of Collaborative Problem Solving. Arthur Graesser, others. Memphis Univ. 2019.

5. Premio Heroes Yahoo Finance 2019.

6. Hugo Alconada Mon, La Nacion. Entrevista. 13/6/2020.

7. Simon (1971). «DESIGNING ORGANIZATIONS FOR AN INFORMATION-RICH WORLD». digitalcollections.library.cmu.edu.

\begin{abstract}
The concept of a global pandemic has forced to change interpersonal communication in an abrupt way. Within this adaptation, the concept of Image as such is developing its reformulation.

Today more than ever Image is perception and communication. In this work you will see how the question about what the Image is has been reformulated almost by itself. As Image specialists, today there appear before us aspects of the lives of others that we did not see before, new needs from the personal image of clients, who ask to incorporate a new learning about their own Image and how it communicates to others.

Today customers perceive that their Personal Branding is key in communication in this new normal. The resignification of the Image concept has initiated a change without return posing a new challenge in the profession.
\end{abstract}

Keywords: New normality - COVID19 - Personal Branding - Personal Brand - Image - Digital - Resilience - Perception - Neuro-leadership - Neurobusiness - Neuroimaging.

Resumo: $\mathrm{O}$ conceito de uma pandemia global forçou a mudar a comunicação interpessoal de forma abrupta. Dentro dessa adaptação, o conceito de imagem como tal está desenvolvendo sua reformulação.

Hoje mais do que nunca Imagem é percepção e comunicação. Neste trabalho, você verá como a pergunta sobre o que é a Imagem foi reformulada quase por si mesma. Como especialistas em imagem, hoje aparecem diante de nós aspectos da vida de outras pessoas 
que não vimos antes, novas necessidades da imagem pessoal dos clientes, que pedem para incorporar um novo aprendizado sobre sua própria imagem e como ela se comunica. Aos demais.

Hoje, os clientes percebem que sua marca pessoal é fundamental na comunicação nesse novo normal. A ressignificação do conceito de imagem iniciou uma mudança sem retorno, colocando um novo desafio na profissão.

Palavras chave: Nova normalidade - COVID19 - Marca pessoal - Marca pessoal - Imagem - Digital - Resiliência - Percepção - Neuro-liderança - Neurobusiness - Neuroimagem.

[Las traducciones de los abstracts fueron supervisadas por el autor de cada artículo] 